\title{
"A Life and Death Situation:" Opportunities for Enhancing Access and Efficacy of Peer Sponsorship in Substance Use Disorder Recovery
}

\author{
Jeremy Heyer, Zachary Schmitt, Lynn Dombrowski, Svetlana Yarosh
}

\begin{abstract}
Substance use disorders (SUDs) are characterized by an inability to decrease a substance use (e.g., alcohol or opioids) despite negative repercussions. SUDs are clinically diagnosable, hazardous, and considered a public health issue. Sponsorship, a specialized type of peer mentorship, is vital in the recovery process and originates from 12-step fellowship programs such as Alcoholics Anonymous (AA) and Narcotics Anonymous (NA). To investigate sponsorship relationship practices and to identify design opportunities for digitally-mediated peer support, we conducted 27 in-depth interviews with members of AA and NA. We identified five key sponsorship relationship practices relevant for designing social computing tools to support sponsorship and recovery: 1) assessing dyadic compatibility, 2) managing sponsorship with or without technology, 3) establishing boundaries, 4) building a peer support network, and 5) managing anonymity. We identify social computing and digitally-mediated design opportunities and implications.
\end{abstract}

\section{Author Keywords}

Substance use disorders, addiction, recovery, 12-step fellowships, technology for substance use, peer health support.

\section{CCS Concepts}

-Human-centered computing $\rightarrow$ Empirical studies in collaborative and social computing;

\section{INTRODUCTION}

In the United States, 20.2 million adults or $8 \%$ of the population suffered from a substance use disorder (SUD) in 2017 [30]. SUDs are defined by the inability to decrease substance use, despite severe negative social, economic, and health-related consequences $[6,65]$ and include a variety of substances $(e . g$., alcohol, opioids, methamphetamines).

\footnotetext{
Permission to make digital or hard copies of all or part of this work for personal or classroom use is granted without fee provided that copies are not made or distributed for profit or commercial advantage and that copies bear this notice and the full citation on the first page. Copyrights for components of this work owned by others than the author(s) must be honored. Abstracting with credit is permitted. To copy otherwise, or republish, to post on servers or to redistribute to lists, requires prior specific permission and/or a fee. Request permissions from permissions@ acm.org.

CHI'20, April 25-30, 2020, Honolulu, HI, USA

(C) 2020 Copyright held by the owner/author(s). Publication rights licensed to ACM ISBN 978-1-4503-6708-0/20/04 . .\$15.00

DOI: https : //doi .org/10.1145/3313831. XXXXXXX
}

A common element in recovery from SUDs is the use of 12step programs such as Alcoholics Anonymous (AA) and Narcotics Anonymous (NA). Development of the sponsor and sponsee relationship is a crucial component of these programs. Sponsors are individuals in recovery who guide others working a recovery program (i.e., sponsees) through the 12-steps by "sharing their experience, strength, and hope." 1

Twelve-step fellowships rely on this form of in-person mentorship to develop healthy recovery practices and support networks. However, forming and maintaining these relationships can be difficult given the negative health outcomes [53], financial difficulties, and cognitive comorbidities [13] that may occur from repeated substance use. Research shows that multiple forms of mentorship and support, such as sponsorship and 12-step programs, significantly increase the potential for continued abstinence or decreased substance use $[63,59,26]$. Despite the importance of sponsorship, few studies have investigated the potential for social computing to support these practices in recovery communities.

In this paper, we report on interviews with 27 members of 12 -step fellowships. We identify and investigate the following research questions:

RQ1. What mentorship practices do sponsors and sponsees adopt to meet the challenges of recovery?

RQ2. How do sponsors and sponsees negotiate divergent needs and motivations?

RQ3. How might we design social computing technologies to support and expand access to sponsorship?

Recovery communities provide unique insights and perspectives on critical threads of prior work in social computing and HCI, including peer support for mental health [40, 20], behavior change [48, 62], and privacy in design [64]. Understanding current sponsorship practices, challenges, and opportunities is essential for developing the next generation of social computing tools for the context of SUD recovery. Our investigation foregrounds five critical priorities for sponsorship: (1) assessing dyadic compatibility, (2) managing sponsorship with or without technology, (3) establishing boundaries, (4) building peer support networks, and (5) managing anonymity.

\footnotetext{
${ }^{1}$ na.org/admin/include/spaw2/uploads.pdf
} 
We begin by providing background information on 12-step sponsorship and relevant prior research. Next, we describe our process and report the findings from our interview investigation. Finally, we present implications for design and future research in this context.

\section{BACKGROUND}

A 2019 estimate identified 1,446,729 AA members in North America and 2,130,419 worldwide, with 67,000 NA members in over 139 countries [54, 38]. There are over 200 different 12step fellowships including AA and NA [65]. Each fellowship focuses on a specific addiction to a substance or behavior (e.g., cocaine, alcohol, overeating, gambling). These fellowships follow a 12-step model that outlines a course of action for recovery [61]. Many of these fellowships also recommend the practice of sponsorship-having more experienced program members guide newer ones through the steps. The following terms are relevant to understanding sponsorship:

- Sponsorship: the practice of a sponsee working with a peer who has more time in recovery (i.e., sponsor) to learn from their experience and be guided through the 12-steps.

- 12-step fellowship meeting: a recovery peer support group that meets regularly (e.g., weekly) to help each other stay clean/sober by sharing their life experiences.

- Step work (i.e., working the steps, homework, and assignments): the specific tasks involved in working the 12 steps, which frequently includes written assignments on topics such as admitting the problem, making amends, and creating a moral inventory of oneself. Step work is done cyclically (i.e., members will complete multiple iterations of the 12-steps). ${ }^{2}$

- Service work: volunteering to provide a variety of support to the fellowship, including cleaning up after meetings, speaking at meetings, and offering to sponsor others.

- Anonymity: members do not identify themselves as members of a particular fellowship in public media and avoid use of titles (e.g., doctor) or last names in 12-step contexts. ${ }^{3}$

Obtaining sponsorship early in recovery has been shown to assist in continued abstinence and long-term sobriety [59, 43]. Sponsorship is not only an important practice in SUD recovery, but also a prototypical form of peer mentorship that may provide new perspectives on a number of research threads in social computing.

\section{RELATED WORK}

We highlight prior work in social computing technologies for dyadic mentorship, peer support in mental health, and SUD recovery.

\section{Social Computing for Dyadic Mentorship}

Recovery sponsorship is a form of dyadic mentorship-a concept investigated in HCI and other scholarship traditions. Traditionally, dyadic mentorship is defined as an intentional

\footnotetext{
${ }^{2}$ https://www.alcohol.org/alcoholics-anonymous/

${ }^{3}$ https://www.aa.org/assets.pdf
}

nurturing process by which the more experienced individual (i.e., the mentor) fosters growth and development in a less experienced protégé $[15,27]$. Prior work shows that dyadic mentorship positively impacts protégés, including youth development in foster care [4], facilitation of career advancement [27], and the reduction of substance use in SUD recovery (i.e., sponsorship) [59]. Dyadic mentoring also benefits the mentor by contributing to a sense of fulfillment and self-efficacy [47].

Beyond face-to-face mentorship, prior research demonstrates the efficacy and challenges of e-mentoring (use of social computing technologies, such as video-conference, email, online networking sites) as the primary connection between a mentor and protégé in a dyadic mentorship relationship [37]. Ementoring sidesteps many of the barriers seen in traditional face-to-face mentorship models that may prevent a potential protégé from obtaining and benefiting from a mentor [5, 46]. Such barriers include a lack of available mentors in an industry, profession, or support community, a lack of time and availability of would be mentors, and unmanageable geographic distance between a would be mentor and protégé.

While e-mentoring sidesteps traditional barriers inherent in face-to-face mentorship, research shows relationship quality may suffer. For instance, Cotton and Adya [14] demonstrate that as dyadic mentoring relationships become more virtual, relationship satisfaction decreases. Similarly, Stone and Lazereski [55] suggest protégés may misunderstand information, have fewer opportunities to clarify advice, and be less receptive to information and advice that mentors provide through electronic media compared to face-to-face communication. Blended online approaches have been suggested to circumvent some challenges associated with face-to-face mentorship while still retaining in-person benefits $[36,7]$.

Prior work in HCI explored the blended mentorship model. For example, Yuan and Yarosh [69] explored design opportunities for technology to support and expand intergenerational dyadic mentorship in schools. However, prior research has not investigated how technology might support and expand access to face-to-face mentorship (i.e., sponsorship) in SUD recovery. Further, prior work demonstrates a strong need for understanding privacy, safety, and other social concerns for blended mentorship [37]. We address these challenges by identifying socio-technical opportunities and risks of blended mentorship for SUD recovery.

\section{Social Computing for Peer Support in Mental Health}

Given that SUDs are frequently categorized as a mental health disorder [6], we examined social computing scholarship concerning peer support for mental health to inform this study.

In-person peer support groups are a common and effective support mechanisms for individuals with mental health disorders because they provide an opportunity to share personal experiences, feelings, coping strategies, and firsthand information about treatment. Participation in in-person peer support groups often reduces re-hospitalization rates, increases engagement in outpatient services, and increases quality of life outcomes (e.g., increased employment rates and reduced depressive symptoms) [2]. Unlike the traditional mentor-protégé 
dyad, peer relationships involve multiple individuals of similar status and experience level and offer a higher degree of mutual assistance, where both individuals give and receive support [27]. Peer relationships have the potential to serve some of the same functions as mentorship, but are often more accessible and available [27].

Online peer support has grown in popularity due to ease of access, flexible participation, and the ability to maintain a degree of privacy $[45,8]$. However, health researchers found that unmoderated online support may have mixed results for people with mental health disorders [34, 19]. HCI research has also sought to characterize these effects by analyzing activity in online communities. For example, De Choudhury and De [16] investigated the language attributes and content that characterize self-disclosure as well as the factors that drive social support in mental health related posts on reddit. Pater et al. [44] analyzed user-generated content regarding eating disorders across several popular social media platforms. Broadly, online support groups or social media use for mental health may need to be used in particular ways to be beneficial, with some evidence that unmoderated online engagement may worsen symptoms [25] and lead to negative experiences such as exposure to misinformation [29].

Recent HCI research sought to mitigate these negative experiences in online peer support by integrating evidence-based strategies. For instance, O'Leary et al. [39] conducted a participatory design study with participants with mental health disorders in order to make mental health support tools accessible, safe, and empowering. Further research by O'Leary attempted to mitigate the negative effects of online peer support by deploying peer support training in the form of a guided chat tool that empowers peers to utilize evidence-based talk therapy strategies [41]. In another example, Morris et al., created Panoply [35], an application to treat depression symptoms by combining evidence-based therapy techniques with peer-to-peer interactions and support.

We build upon this body of work, but rather than focusing on exclusively online peer support, we explore how technology can leverage existing face-to-face peer support networks and social structures of 12-step fellowships.

\section{Substance Use Disorder Recovery in $\mathrm{HCl}$}

HCI scholarship has investigated substance use and recovery using computational methods to detect and predict behavior and applied design-focused investigations to better understand people's technology preferences and concerns for SUD recovery.

Within detection, computational research has focused on detecting substance use with mobile phone sensors for breath [68], saliva [66], and walking gait [24]. These technologies allow monitoring substance use following acute treatment. In the space of prediction, HCI research has used computational methods to categorize and predict relapse, recovery, and treatment factors based on language used in social media and peer support forums dedicated to substance use recovery $[11,31$, $56,57]$.
Most relevant to this work, HCI researchers have conducted design-focused investigations to better understand how technology can support individuals in recovery from SUDs. This has included formative work to explore how technology can be integrated into established recovery traditions and practices $[65,51,52]$ and the development of specific technologies to support family communication [67], suggest relapse prevention skills [68], and explore the efficacy of virtual counseling agents [70].

In this paper, we explicitly investigate opportunities for technology-mediated sponsorship. Prior work has identified sponsorship as a potentially fruitful area for socio-technical intervention [52]. However, prior work did not specifically explore which aspects of such interventions would be acceptable to the recovery community and did not include perspectives of potential sponsors. Our paper contributes to these ongoing discussions by identifying sponsors and sponsees's specific recovery needs, priorities, and strategies, and by identifying opportunities and risks for socio-technical interventions in this context.

\section{METHODS}

We used in-depth interviews to characterize opportunities and risks for socio-technical support for sponsorship.

\section{Participants}

We interviewed 12 sponsees (see Table 1) and 15 sponsors (see Table 2) who identified as members of one or more 12step fellowships regarding their experiences with providing or receiving sponsorship. All sponsee participants were in an active relationship with a sponsor. Sponsor participants were either in an active relationship with one or more sponsees or had prior experience providing sponsorship. Participant sponsees and sponsors were not affiliated with each other (i.e., we interviewed individuals, not dyadic pairs).

We began by recruiting seven sponsee participants through their affiliation with an all-female recovery home in [location redacted] with which we had an existing research partnership. We then continued "abductive sampling" [42] to include experiences and perspectives which may not have been represented in this original set. As the recovery home mostly included people who were receiving rather than providing sponsorship, our first abductive sampling priority was including the perspective of sponsors. We recruited 15 sponsor participants through word of mouth and through a local recovery certification agency in [location redacted]. Next, we sought to diversify the gender balance of our sponsee sample by recruiting three male participants affiliated with an all-male recovery home in [location redacted]. Finally, we sought to recruit participants with more long-term experience being a sponsee, as most of our initial participants were relatively new to recovery. Thus, we recruited two participants with more than a decade of experience being a sponsee through their affiliation with an addiction intervention center in [location redacted].

\section{Ethical Consideration for Anonymity}

We took several steps to safeguard participant anonymity during the study. Since many of the participants recruited were 


\begin{tabular}{cccc}
\hline P\# & Gender & Recovery Time & Fellowship \\
\hline P1 & F & 5 months & AA \\
P2 & F & 8 months & AA, NA \\
P3 & F & 7 months & AA \\
P4 & F & 9 years & AA \\
P5 & F & 13 months & AA, NA \\
P6 & F & 8 months & AA \\
P7 & F & 12 months & NA \\
P8 & M & 7 months & AA, NA, HA \\
P9 & M & 2 years & NA \\
P10 & M & 1 months & AA \\
P11 & F & 19 years & AA \\
P12 & M & 15 years & AA, NA \\
\hline
\end{tabular}

Table 1. Sponsee demographics and fellowships. Fellowships included: AA, NA, HA (Heroin Anon.).

\begin{tabular}{cccc}
\hline P\# & Gender & Time as Sponsor & Fellowship \\
\hline P13 & M & 6.5 years & AA \\
P14 & F & 6 years & AA, NA \\
P15 & F & 5 years & AA, CA \\
P16 & F & 10 years & NA \\
P17 & F & 20 years & AA \\
P18 & F & 16 years & NA \\
P19 & M & 3 years & AA, NA \\
P20 & M & 6 months & NA \\
P21 & F & 4 years & AA \\
P22 & M & 5 years & NA \\
P23 & F & 23 years & AA \\
P24 & F & 10 years & AA \\
P25 & F & 4 years & AA \\
P26 & F & 22 years & AA \\
P27 & F & 10 years & AA \\
\hline
\end{tabular}

Table 2. Sponsor demographics and fellowships. Fellowships included: AA, NA, CA (Cocaine Anon.).

affiliated with the same institution (e.g., a specific recovery facility, sober home, meeting), we wanted to ensure that participants could remain anonymous even if a specific participant from an institution disclosed their own study participation. To do so, we chose not to collect or report participant demographic data (e.g., age, drug of choice), which may otherwise allow identifying a specific individual within an institution. To respect 12-step fellowships' tradition of anonymity, we received permission from the IRB to waive documentation of consent and permission from our departments waive collecting signatures confirming compensation. By removing the requirements for documenting consent and compensation, we circumvented the need for participants to provide their name at any point in the process.

\section{Procedure}

We used email or phone to set up a time and place for the interviews. Fourteen interviews were conducted in-person (seven sponsors and seven sponsees) at a location of the participant's choosing, such as a coffee shop or their home. The remaining thirteen interviews were conducted over the phone, with con- sent information forms emailed to the participants prior to the call.

After verbally confirming informed consent, each interview lasted between 45 and 90 minutes. Interviews were semistructured, allowing participants to drive the conversation. We began by discussing the participant's background, history in recovery, and relevant experiences either receiving (for sponsees) or providing (for sponsors) sponsorship. Common topics across interviews including experiences initiating and maintaining a sponsorship relationship, challenges faced and strategies used (including current technology use), and perceived priorities and concerns in the potential use of technology to augment current sponsorship practices. After completing the interview, participants were compensated with a fifteen-dollar gift card.

\section{Analysis}

Each interview was audio recorded, transcribed, and analyzed by the interview leads from each site. We used inductive thematic analysis characterized by the generation and constant comparison of open codes in order to reveal underlying themes (e.g., $[12,42])$. We began analysis by performing line-by-line open coding and memoing on the qualitative data collected from each interview, identifying short, individual units of meaning within the textual data [12]. We used the process of affinity diagramming to spatially cluster open codes based on similar meaning [42]. Following this initial analysis, the research team came together to discuss the preliminary findings and thematic code clusters from the sponsor and sponsee data sets. We then iteratively refined and combined these clusters to identify five recurring and consistent themes across the sponsor and sponsee participants.

\section{RESULTS}

Through thematic analysis of sponsor and sponsee interviews, we identified five sponsorship practices that characterized the relationship between sponsors and sponsees. 1) assessing dyadic compatibility, 2) managing sponsorship with or without technology, 3) establishing boundaries, 4) building peer support networks, and 5) managing anonymity.

\section{Assessing Dyadic Compatibility}

For sponsees and sponsors, dyadic compatibility served as a foundational building block of sponsorship. Dyadic compatibility is the shared understanding that the mentoring relationship is likely to be successful, effective, and viable based on mutual assessment of "fit." However, sponsors and sponsees valued different aspects of the relationship when assessing dyadic compatibility. Sponsees assessed their sponsors to understand if they had similar or complementing personality traits and recovery experiences. Whereas sponsors assessed dyadic compatibility in terms of common expectations and goals for the relationship.

\section{Sponsees Value Compatible Personality and Experience} Sponsees are responsible for identifying and initiating contact with a potential sponsor, so sponsees were able to articulate a number of issues they considered in deciding whom to ask. Sponsees stated that an ideal sponsor is a person with whom 
they can be "completely vulnerable, and honest" (P2) and "if you do fall down and relapse, they are gonna be there to pick you up and not judge" (P4). Sponsee P6 stated that finding a sponsor with whom she felt comfortable with was essential for open communication. "You need that comfort level with your sponsor so you feel like you can open up to them about things. Because if you're afraid to talk to them about what is really going on, then you are not benefiting from it."

Sponsees often used the in-person 12-step fellowship meetings to connect with and assess a potential sponsor's personality, recovery background, and interaction style. For instance, sponsee P4 stated that she "listens to others at meetings and I look for somebody that either says something that was important to me or they seem to have qualities that I would like to have" when searching for a sponsor. This method provides her with a "gut feeling" regarding which individual present at the 12-step fellowship meeting might be a compatible sponsor.

For sponsee P1, a 12-step fellowship meeting provided an opportunity to identify a sponsor with whom she established a successful sponsorship relationship. "I just liked her personality. She was really charismatic and artistic and after spending time with her and seeing how she interacts with people, that was just how I wanted to interact with people." In this case, a brief introduction at a 12-step fellowship meeting was sufficient to assess compatibility with a potential sponsor.

However, while some sponsees were able to get a "gut feeling" (P4) regarding a potential sponsor, the 12-step fellowship meeting format may not provide sufficient information to gauge sponsor fit. For instance, sponsee P6 stated that political ideology, a topic rarely discussed at 12-step fellowship meetings, is vital to compatibility. "I don't want to work with the right wing conservative, like that is just not something I would be comfortable with. That is just important to me.” Differences in political ideology led to the end of the sponsorship relationship, forcing sponsee P6 to restart her search for a sponsor. Similarly, sponsee P12 stated that he struggled working with a sponsor whose experiences were too different from his own. “We just didn't click completely. I did a lot drugs, but he only drank, I spent time in prison, he didn't. Our viewpoints were different. I couldn't connect and wasn't completely honest with him." In this case, the lack of shared experience led to an incompatible match and an unwillingness for sponsee P12 to be open and honest with his sponsor.

Throughout our interviews, sponsees were able to articulate the type of relationship they wanted with their ideal sponsor, but discussed many difficulties and false starts in their attempts to find and initiate that ideal relationship. Sponsees are faced with reaching out to potential sponsors which can be a daunting task for somebody who is new to a 12 -step fellowship. For instance, sponsee P5 stated, "I think I had a lot of fears in finding a sponsor. Like what if I don't pick the right one? What if I let this person down? I think it was hard for me to pick somebody." From the perspective of the sponsee, choosing the right sponsor will have a large impact on their recovery. In an attempt to choose the right sponsor to support their recovery, they assess the compatibility of a sponsor based upon personality, life experience, and recovery experience.

\section{Sponsors Value Compatible Relationship Expectations}

A person who is asked to become a sponsor may agree or reject the offer either immediately or after a trial period of evaluating the sponsorship relationship. While a small number of sponsors agreed that similar personality and life experiences were valuable components of a successful relationship, the majority of sponsors focused more heavily on the value of compatible expectations. All 15 sponsor participants emphasized that their primary expectation was that a sponsee display a consistent "willingness"to commit to the recovery program and be open to the sponsors' suggestions. Only by meeting this expectation of willingness, were sponsors prepared to fully devote their time and energy to help a sponsee. "Willingness requires that a sponsee be willing to work their recovery. I'm not going to carry somebody through recovery...but if they are willing and they want to get sober, then by all means I'd love to help them do that"(P20). Sponsors focused on setting up clear relationship expectations and roles to foster a successful sponsorship experience. Sponsors often established expectations early in the relationship through in-person meetings that take place prior to starting other activities together.

\section{Managing Sponsorship with and without Technology}

For sponsors and sponsees, opportunities to meet in-person (either one-on-one or as part of 12-step fellowship meetings) are a critical part of developing a recovery relationship. Shared 12-step fellowship meetings offer opportunities to collectively share experiences "honestly" (Sponsor 14) with others in the recovery community. A typical expectation set by sponsors is that sponsees attend some 12-step fellowship meetings together. Sponsor P17 explains why she attends a specific 12 -step fellowship meeting as context for suggesting that her sponsee do the same: "I go to a Monday night [12-step fellowship meeting], it is not my favorite meeting but that is where my sponsor is, and I wanna keep my sponsor so that is why I go." Aside from shared 12-step fellowship meetings, participants also discussed the importance of regularly meeting one-on-one to discuss progress and step work, which allows them to strengthen the sponsorship bond by building trust, discussing challenges, evaluating progress, and connecting over shared life experiences. Sponsors expressed the expectation that sponsees regularly schedule these opportunities to connect.

The value of one-on-one meetings was often discussed in contrast to having the same conversations mediated via phone, SMS, or other computer mediated tools. For example, sponsee P6 discussed how being in-person allowed her to open up more: "I am able to open up to her [sponsor] in person, and she kind of... will give me similar situations that she has been through, it makes me feel less alone, which is cool." Most sponsor and sponsee participants acknowledged how one-on-one meetings further deepen the sponsorship relationship because it requires actual physical presence which allows for a deeper connection. As sponsor P21 emphasized: "just being physically present, giving them a hug can be very important."

However, despite recognizing the drawbacks of mediated communication, sponsors admitted to opportunistically using various digital communication tools to supplement functions of 
the sponsorship relationship. Some of this tool use included texting daily to check-in (P14) or using online chat rooms to seek help (P27). Others used tools to simplify the logistical aspects of working together such as using online websites to help schedule a meeting time (P13), sending literature through Snapchat (P16), and sharing PDFs of step work assignments (P24). During temporary times of geographic separation, sponsors discussed using ecosystems of tools to connect, for example sponsor P27 described: "I got a sponsee right now that is out of town and she texts me pictures of her worksheets and ... we've been reading over the phone together..."

There were divergent perspectives on whether and how often technology should be used. Sponsor P22 chalked it up to a compatibility of expectations: "if you just want to text and email as a form of doing this [sponsorship], I am not your guy." Some sponsees also felt that current trends for turning aspects of one's personal life to mobile apps would trivialize and dehumanize recovery: "I think it depersonalizes something that is very personal in someones life. For the most part, this is a life and death situation. So, to put that on a phone app, I am not in agreement with that" (P10). However, the majority of our participants reported adopting one or more technologies to aid in various aspects of recovery (e.g., an app to find NA meetings, a podcast of AA speakers).

While wary of replacing in-person contact with technology, sponsor and sponsee participants recognized that a variety of information and communication technologies could augment supportive components of the sponsorship relationship (i.e., attending meetings, homework assignments, and check-ins). Overall, traditional communication methods are preferred, but there is a growing number of sponsors and sponsees open to complementing traditional methods with technological approaches.

\section{Establishing Boundaries}

For sponsor participants, creating and affirming boundaries were integral components of their sponsorship practice. Boundaries provide several key functions for the sponsor relationship, including creating implicit and explicit rules about behavior and modeling a healthy relationship for the sponsee. Boundaries often revolve around timing, type of communication, and support exchanged between a sponsor and sponsee. Sponsor P17 explained why she sets boundaries when developing the relationship: "You have to set [boundaries] to make sure [sponsees] do not become dependent on you...because if they do and a [sponsor] leaves or dies then guess what? [The sponsees] are gonna go drink or [do] drug[s]."Sponsors reported trying to set boundaries that encourage sponsees to take responsibility for their own recovery, as sponsor P20 emphasized: "I'm not gonna drag 'em [through their recovery]."

This sponsor priority at times conflicted with the stated needs of sponsees, who expressed a greater need for accountability structures and guidance in decision making. Most participants reported poor decision making during addiction and some sponsees discussed seeking out sponsors that could guide them in healthy decision making and offer a rigid structure in recovery. For example, sponsee P2 stated she sought a sponsor that would "ride [her] ass", to ensure compliance with her sponsor's requests, while sponsee P4 explained how she struggled with a "super gentle" sponsor and now seeks sponsorship with a sponsor that would, "hold me accountable and call or text me." Most sponsees identified accountability and reliability as sought after characteristics for sponsorship, while most sponsors emphasized personal willingness and responsibility.

Boundaries have additional importance for sponsors, as they enable sponsors to create time for their own recovery and lives. All sponsors identified boundaries as rules that allow them to have social and emotional time away from their sponsorship relationships. Sponsor P18 expressed that she sets boundaries with her sponsees because of the many diverse roles she plays in the recovery process. "I set boundaries for my self-care. Especially because I am a recovery coach, a member of narcotics anonymous, and a sponsor." This concept of self-care or self-preservation is rooted in the idea that a sponsor must have the capacity to give back. As sponsor P26 stated, "there is a piece [of me] that has to take care of myself in order to take care of anyone else." This element of boundaries as self-preservation may be particularly critical when a sponsee relapses, as sponsor P17 discussed: "You see them going south, right? And here's what I say. You are on the edge of the cliff and what I am gonna ask you to do is take two steps back. I am not gonna stand there on the cliff with you because if you fall over I am gonna try to grab you and you will pull me over." Sponsors must protect their own recovery before they can help others.

While recognizing the importance of consistent boundaries, most sponsors acknowledged that their boundaries varied depending on the sponsee's needs and recovery progress, as sponsor P26 explained: "The way I walk through the steps does not change. But each of my sponsees is different and they need something different from me. And I have got to identify what that is." For many of the sponsors, their established boundaries or rules became more flexible during moments of crisis, such as a relapse or a deterioration after improvement, due to the severe emotional, mental, and physical strain that often accompanies the first few weeks of recovery after relapse. Sponsor P15 discussed how in early recovery she often does not place a limit or boundary on communication or contact. "Very early on we try to be as available as we can. So you try to answer as much as you can in the beginning." Sponsors must evaluate their capacity to provide support and their sponsees' needs on a case-by-case context in this high-stakes context.

Boundaries are used by sponsors to provide sponsees with healthy models of relationships and to decrease co-dependence. Boundaries need to be versatile to the sponsee's individual needs while still prioritizing the sponsor's own recovery. The gap between what sponsees may want and what their sponsor may be able provide characterizes one of the core tensions of the current sponsorship model.

\section{Building Peer Support Networks}

Individuals in recovery often have unpredictable and complicated needs that cannot be fully addressed by just their sponsor. For that reason, sponsors encourage sponsees to form a robust and trusted peer support network to supplement sponsorship. In this section, we explore how peer support occurs and how 
sponsors and sponsees build these potentially lifesaving peer support networks. We also discuss how sponsees and sponsors develop peer support networks to help during crisis moments and to find support beyond individual sponsor's expertise.

\section{Building Peer Support Networks Via Sponsorship Family}

The sponsor often helps the sponsee build a peer support network for their recovery. A sponsor often connects sponsees to their larger "sponsorship family" (i.e. their sponsor's sponsor and their other sponsees), which adds trusted individuals to the sponsee's social support network. As sponsee P5 describes: "One thing I really like about my sponsor is that we kind of have a few women to lean on that are also in the sponsorship family, I have my sponsee sisters, so you know if I can't get an answer from [my sponsor], I can also go to my sponsee sisters.” The sponsorship family provided sponsees with a vetted peer group that was close in geographic proximity, and attended many of the same 12-step fellowship meetings - which provides additional opportunities to strengthen bonds.

\section{Urgent, Time-sensitive Support}

Sponsees discussed how stress, restlessness, and other relapse triggers come about suddenly and unpredictably, especially in early recovery. Timely trusted social support helps manage these situations and avoid relapse. Given that a single sponsor cannot provide social support 24 hours a day, sponsees often relied on their peer networks to provide them with support when their sponsor was unavailable. For instance, sponsee P2 discussed how she reaches out for support in times of need. "I would try and call my sponsor first, but she has a life too, I'm not expecting her to answer right away, I have a lot of other people I can call, which is awesome." Sponsors encourage sponsees to have multiple people to rely on in an emergency situation, as sponsor P14 describes: "I encourage people [to connect with others when she is unavailable]. My phones off when I go to bed ... if you call the first person and they do not pick up, keep calling other people. Somebody will pick up." For sponsees, a peer support network helps overcome periods of distress or cravings, especially when a sponsor is unavailable.

\section{Support Needed Beyond the Sponsor's Experience}

Beyond the urgent and time-sensitive support, peer support helps fill-in gaps in the sponsor's experience when sponsees need specific guidance. As sponsors are tasked with sharing only about their own "experience, strength, and hope," 4 there may be times when they need to refer the sponsee to others in their network for specific guidance. For example, when asked for childcare guidance, sponsor P14 shared their approach: "if you need single mom help, let's go find you some single moms to talk to." Similarly, sponsor P17 discussed a hypothetical situation regarding how to support a sponsee that is grieving a family death: "We just call somebody that has experience, strength, and hope in what you're going through....ya know [redacted name] lost her mom two years ago, honey you may want to call her." A diverse peer support network with a wide spectrum of life experiences is a vital resource for sponsors to connect their sponsees with necessary support-especially

\footnotetext{
${ }^{4}$ na.org/admin/include/spaw2/uploads.pdf
}

when sponsors themselves does not have experience with a particular topic.

\section{Managing Anonymity}

"Anonymity is the spiritual foundation" of all 12-step fellowship traditions [1]. Prior work examines how 12-step communities strive to preserve anonymity with digital technologies $[50,65]$ and found three critical ways the group strives to protect anonymity. First, these groups protect people's privacy by having strict rules regarding disclosures of private information, including not revealing who is in recovery nor what is said in meetings [50]. Second, anonymity means that people do not publicly identify as members of a particular 12-step fellowship, which helps protect the program's reputation from any individual's misconduct [50]. Third, anonymity is interpreted as a quality of "namelessness" which is used to emphasize that all members are equal-this definition diverges from the conventional definition of "anonymity" but is critical in the context of 12-step fellowships [50]. Prior work examining digital technologies in this context suggest that individuals in recovery are reticent to use technology that implements a persistent digital identity as it may conflict with this desire for equality through "namelessness" [65]. We examine our participant's anonymity preferences and the tensions that result when anonymity needs directly conflict with sponsorship's social priorities.

\section{Anonymity and Managing Stigma}

Stigma associated with SUDs has the potential to severely and negatively impact employment, social relationships, and even custody of children. As sponsor P23 shared about her early experiences: "I was absolutely mortified when I first got sober. Somebody was gonna find out I was in AA and then how would they look at me professionally." Anonymity helps participants and others manage social stigma by keeping their recovery status private outside of 12-step contexts. Helping protect others' anonymity is considered an important priority: "I am big on everyone else having their own anonymity, so I don't want to break someone else's anonymity" (P19). In alignment with prior work in the 12-step context [50], participants emphasized the importance of being able to control when and how they break their anonymity.

However, our interviews revealed that one element of managing stigma was unique to the sponsorship context, in that concerns about stigma applied not just in relating to the outside world, but also in protecting individuals from stigma within the program. As part of working the steps, sponsees disclose information to their sponsor that they may not share with others, including close ties or others in recovery. Sponsee P7 maintains that "[a sponsor must be] someone I can trust not to divulge shit that I have done, like whatever indiscretions, secrets, crimes, and dark thoughts to other people."Sponsee P6 also emphasized the importance of "protecting anonymity and the privacy of the conversations that you have with a sponsor, so not everybody in the whole program will have access to that." For a sponsor, this may mean treading carefully when seeking additional support for their sponsees, since some experiences may be revealed in confidence. 


\section{Identity Disclosures are Necessary}

While sponsors emphasized protecting others' anonymity, many of them described being willing to disclose their own recovery status to help others. For instance, sponsor P17 recounted an experience where she revealed she was in recovery to someone struggling with substance use: "I revealed who I was because I knew she had a problem. I introduced her to the program and started taking her to meetings..." Self-disclosure of one's own recovery status is necessary in order to make oneself available as a sponsor or as a sponsee. In conventional 12-step arrangements, this need to maintain anonymity outside of the program while making yourself available to give and receive support within is managed through the social contract of 12-step meeting attendance-meetings can be seen as safe spaces to disclose recovery status. Sponsor P22 discussed the importance of similar "addicts-only" spaces online: "Yeah, addicts only ... until we prove you are, you can't get on the page." Sponsees were also enthusiastic about some kind of access control: "make sure that it's not just like Joe-Schmoe joining. [Otherwise it's] like Tinder ... you don't want your face to be like, boom" (P7). Future technologies will have to identify strategies to enable disclosing recovery status while protecting anonymity.

\section{Anonymity Conflicts with Individual Safety}

Prior work argues for ephemeral identities in online recovery spaces to support the qualities of "namelessness" and equality as interpretations of anonymity in 12-step fellowships [65]. However, our interviews revealed a divergent set of priorities introduced by the specifics of the sponsorship context. Unlike general recovery activities, which happen in public spaces with multiple participants, sponsorship inherently requires one-onone work with some degree of privacy. This increases the potential risks to an individual's safety, so many participants described a need for persistent, verifiable identities to protect members from predatory behavior. Unlike other 12-step contexts, where the dominant message to disruptive members is to "keep coming back," in the context of sponsorship participants wanted to, "be able to report harassment ... if someone gets reported several times, they get kicked out... and can't join again" (P2) and were willing to have "the system [moderators] handle ... the guidelines and boundaries ... to show that we are safe using this app." Participants also wanted another layer of safety by only gradually revealing information that could affect personal safety (e.g., phone, address, photo). For example, one sponsee liked how in-app messaging could remove the need to share a phone number: "...that way they don't have my phone number ... and from there, I can decide if I want to give them my phone number or not" (P5). Another sponsee mentioned waiting until "after you make that first initial contact ... to exchange photos" (P7). Each of these ideas point to the importance of a persistent online identity in tools that attempt to support sponsorship - a perspective that may be at odds with other areas of recovery.

\section{DISCUSSION: IMPLICATIONS FOR DESIGN}

Based on our interview findings, we identify three critical opportunities for socio-technical interventions to support sponsorship in recovery communities: (1) develop sponsors' capacity, (2) facilitate sponsorship initiation, and (3) grow the support community for sponsees. We expand on each of these opportunities based on our findings.

\section{Developing Sponsors' Capacity}

Prior investigations by Witbrodt et al., [63] and Tonigan [59] found that the earlier a sponsee finds a sponsor the more likely they are to maintain continued abstinence. In 12-step fellowships, sponsorship is critical to recovery programs. In "Assessing Dyadic Compatibility," sponsees discussed difficulties of finding compatible sponsors or staying with less effective sponsors due to the lack of available sponsors. In "Establishing Boundaries," sponsors shared being overwhelmed with responsibilities of helping sponsees while also managing their own recovery. Therefore, a key opportunity for technology is to develop sponsors' capacity to meaningfully support multiple sponsees.

In-person sponsorship is irreplaceable in recovery and should only be supplemented with mediated communication. In "Managing Sponsorship with and without Technology," participants worried about losing the deep and meaningful bonds that promote closeness, confidentiality, and familiarity developed during in-person meetings and would not be open to mediating such recovery aspects. While computer-mediated communication does provide opportunities to expand access to one's sponsor when temporarily geographically-separated, such technologies should be used sparingly in the sponsorship context.

However, this does not mean that technology cannot develop sponsors' capacity. Technology could play a key role in supporting daily sponsorship logistics, such as scheduling one-on-one meetings, receiving step work assignments, and following up with sponsees. In "Managing Sponsorship with and without Technology," some sponsors and sponsees shared the complex ecology of tools that they employed to support their practices-step work assignments were shared using photo-sharing apps, check-ins handled via text, meetings scheduled using calendar applications. In "Establishing Boundaries," sponsees shared the need for more regular followups to hold them accountable for their recovery. Facilitating these routine recovery activities is a clear opportunity for mobile applications or virtual assistants.

In "Establishing Boundaries" and "Building Peer Support Networks," our findings highlight the challenges sponsors face in helping sponsees. A sponsee may want more support than sponsors can reasonably offer. Sponsees may experience crises that sponsors may not have the experience, time, or emotional capacity to handle while protecting their own recovery. Sponsees often need to connect with people who have relevant experiences beyond their sponsor. This points to an opportunity to create spaces for sponsors to exchange support and sponsorship strategies to grow their capacity to meet these challenging needs. Health informatics research focuses on supporting caregivers $[9,22]$ and adapting similar strategies to "help the helpers" may help sponsors be more effective both in their own recovery and as sponsee support. One challenge for technology designers will be providing such a support space while respecting the need for absolute confidentiality in the sponsorship relationship revealed in "Managing Anonymity". 
Solutions may range from social (e.g., providing sponsors with guidance on how to seek advice without breaking sponsee anonymity) to technical (e.g., soliciting sponsees to assent before posting any specific message that pertains to them).

\section{Facilitating Sponsorship Initiation}

In "Assessing Dyadic Compatibility," sponsees expressed significant anxiety associated with finding the right sponsor while sponsors expressed frustrations with divergent expectations in the sponsorship relationship. Previous mentorship research identifies how a similar fit between a mentor and protégé can lead to an enduring and effective relationship [28]. Conversely, our work revealed how a lack of fit between a sponsor and sponsee can lead to an ineffective sponsorship experiences or the dissolution of that relationship. These "false starts" are costly to the recovery community, both in terms of reduced sponsor capacity (i.e., a sponsor in an incompatible relationships is not available to take on a more compatible sponsee) and in terms of the increased relapse risks faced by sponsees who do not get needed support. There is an opportunity for technology to facilitate the sponsorship initiation process.

A design opportunity is to expand the sponsees' pool of potential sponsors and reduce false starts by identifying compatible nearby sponsors. In "Assessing Dyadic Compatibility," we discussed aspects sponsees of a potential sponsor that may influence compatibility, including prior experiences, demographics, and religion or political views. Such aspects could easily be provided as elements of a sponsor's online profile and used to filter potentially incompatible sponsors, thus reducing sponsorship "false starts." Personality was also considered as important by sponsees, and was typically assessed by observing the potential sponsor at 12-step meetings. However, if a sponsee regularly attends specific meetings, they may only be exposed to a small portion of the available sponsors in a geographic area. A technologically-mediated sponsorship directory could allow sponsees to identify and attend nearby meetings with potentially compatible available sponsors. Facilitating mutual meeting attendance rather than digitally connecting the sponsor and sponsee, capitalizes on the priorities for in-person connection that we identified in "Managing Sponsorship with and without Technology."

While on the surface, creating any kind of sponsor directory may appear to conflict with the priorities of managing anonymity in recovery, our findings in "Managing Anonymity" point to some compelling alternative perspectives. We found that many sponsors are willing to reveal their recovery status in protected (e.g., "addict-only") spaces. To create protected recovery-only digital spaces and directories, we can leverage the existing social contracts associated with physical attendance at 12-step meetings. So for example, access to a local sponsors directory may be distributed as a printed card at meeting locations or may only be available to people whose phone location identifies them to be at a 12-step meeting location a certain number of times. Even with these protections, participants also preferred that personal information (particularly, phone, address, or photo) is revealed gradually rather than by default and that sponsorship lists be moderated as issues of personal safety may be particularly salient in the sponsorship context.

\section{Growing Sponsees' Support Community}

In "Establishing Boundaries" and "Building Peer Support Networks," our findings reveal that sponsors and sponsees rely on peer support networks to provide complementary support to the sponsorship relationship. Sponsors encourage sponsees to develop robust peer support to foster independence and multiple support avenues. Dependence on a sole sponsor is unhealthy and unsustainable for sponsors and could leave a sponsee without adequate support if a sponsor were to move, pass away, or relapse. Peer networks provide support during crisis moments (when a sponsor was unavailable) and help diversify their support base beyond their individuals sponsor's expertise. Thus, we recommend that technology design assist individuals in recovery by building vetted diverse peer support networks to complement existing support.

One opportunity in this design space is informed by prior work: identifying nearby non-substance-users to help sponsees grow their recovery network by making new clean/sober friends. Zywiak et. al., [71] found that peer support is only beneficial to SUD recovery if a peer network has a higher proportion of individuals who do not use substances compared to individuals who use substances. Peer networks with more substance users than non-substance users are not beneficial, and can negatively influence treatment outcomes [32]. Furthermore, the size of the daily peer support network (i.e., the number of people that an individual in recovery interacts with on a daily basis) is negatively correlated with relapse rates [71]. In other words, a large peer network is more beneficial than a small peer network, but only if frequent interaction occurs. Thus, we propose that future technology design assist individuals in recovery in finding and forming bonds with non-substance-users who live in close geographic proximity and help identify opportunities for in-person interaction.

Another clear opportunity in this space is connecting sponsees with their broader sponsorship family so they have more places to seek support. Participants in this study often cited their "sponsorship family" (i.e., their sponsor's sponsor and other sponsees) as a reliable and meaningful source for peer support relationships. Future design may focus on providing individuals with additional opportunities to meet and interact with members of their "sponsorship family" as it consists of individuals who meet the criteria of a beneficial peer support network (i.e., non-substance users who generally live in close physical proximity). For example, technology that alerts or encourages individuals to attend an event, recovery meeting, or casual meet-up that is being attended by members of their extended sponsorship family may provide additional opportunities to connect with this valuable peer group. However, based on our findings in "Managing Anonymity" it is critical that such a system be designed with "opt-in" functionality allowing each person to set the degrees of separation for their visibility to the network.

\section{LIMITATIONS AND FUTURE WORK}

First, while in-depth interviews enabled us to investigate sponsors' and sponsees' experiences, values, and priorities, indepth qualitative interview methods allows for transferability but not generalization. Therefore, our findings will need to 
be evaluated using other more systematic methods, such as large-scale surveys.

Second, 19 of our 27 participants were women, presenting a gender bias. This gender imbalance is not atypical, as women are more likely to volunteer to participate in research studies $[49,58]$. As a benefit, this imbalance allowed us to focus more on the needs and priorities of women in recovery, an oftenoverlooked population in SUD research [60], but future work should investigate the perspectives of men and non-binary people in recovery.

Finally, our study focused on mentorship in 12-step recovery, but this approach may be considered controversial. Detractors cite the core religious elements of the program [10] as an argument for alternative evidence-based approaches (e.g., SMART Recovery [21], Cognitive Behavioral Therapy (CBT) [33]). However, we found it valuable to focus on 12-step recovery, because this approach is the most widely used form of long-term maintenance and social support for individuals with SUDs $[17,18]$. Prior work indicates that 12-step programs contribute to positive recovery outcomes [23] and are recommended as a best practice by the NIH [3]. Due to our focus on 12-step recovery, some of our findings may be specific to the practices in these programs (e.g., "managing anonymity"), while others (e.g., establishing boundaries, building peer support networks) may apply to SUD peer support more broadly. Further investigations with other forms of peer support may help articulate the generalizability of these findings. Despite these limitations, we believe our work provides valuable insight on how design may support and expand access to peer mentorship in SUD recovery.

\section{CONCLUSION}

Members of 12-step fellowships leverage the practice of sponsorship to support each other in recovery from SUDs. We conducted interviews with 12 sponsees and 15 sponsors to inform design recommendations to support these critical relationships. Sponsors and sponsees had divergent priorities when assessing compatibility and setting boundaries. While sponsors established boundaries to protect their recovery and discourage codependence, sponsees wanted and sought additional support through their broader recovery community. We found that participants engaged in frequent in-person meetings with their sponsor were wary of mediated communication. Participants were vigilant in protecting others' anonymity to mitigate SUD stigma, but could chose to break their own anonymity to make themselves available to help others. We offer specific design recommendations to increase sponsor capacity, facilitate sponsorship initiation, and grow a broader support community for sponsees.

\section{REFERENCES}

[1] 1953. Twelve steps and twelve traditions. ([1st ed.].. ed.). Alcoholics Anonymous Publishing, inc., New York.

[2] 2019. Evidence for Peer Support. Technical Report. Mental Health America. 9 pages.

https://mhanational.org/sites/default/files/ Evidence20for\%20Peer\%20Support\%20May\%202018.pdf
[3] National Institute on Drug Abuse. 2018. Treatment and Recovery. (2018).

https://www.drugabuse.gov/publications/

drugs-brains-behavior-science-addiction/

treatment-recovery

[4] Kym R. Ahrens, David Lane DuBois, Laura P. Richardson, Ming-Yu Fan, and Paula Lozano. 2008. Youth in foster care with adult mentors during adolescence have improved adult outcomes. Pediatrics 121, 2 (2008), e246-e252.

[5] Tammy D. Allen, Mark L. Poteet, and Susan M. Burroughs. 1997. The mentor's perspective: A qualitative inquiry and future research agenda. Journal of vocational behavior 51, 1 (1997), 70-89.

[6] American Psychiatric Association. 2013. Diagnostic and statistical manual of mental disorders (DSM-5Âö). American Psychiatric Pub.

[7] EunJin Bang and Julie A. Luft. 2013. Secondary science teachers' use of technology in the classroom during their first 5 years. Journal of Digital Learning in Teacher Education 29, 4 (2013), 118-126.

[8] Gary G. Bennett and Russell E. Glasgow. 2009. The delivery of public health interventions via the Internet: actualizing their potential. Annual review of public health 30 (2009), 273-292.

[9] LMM Boots, ME De Vugt, RJM Van Knippenberg, GIJM Kempen, and FRJ Verhey. 2014. A systematic review of Internet-based supportive interventions for caregivers of patients with dementia. International journal of geriatric psychiatry 29, 4 (2014), 331-344.

[10] Charles Bufe. 1997. Alcoholics Anonymous: cult or cure? See Sharp Press.

[11] Stevie Chancellor, George Nitzburg, Andrea Hu, Francisco Zampieri, and Munmun De Choudhury. 2019. Discovering alternative treatments for opioid use recovery using social media. In Proceedings of the 2019 CHI Conference on Human Factors in Computing Systems. ACM, 124.

[12] Kathy Charmaz and Linda Liska Belgrave. 2007. Grounded theory. The Blackwell encyclopedia of sociology (2007).

[13] Domenic A Ciraulo, Joanna Piechniczek-Buczek, and E Nalan Iscan. 2003. Outcome predictors in substance use disorders. Psychiatric Clinics of North America (2003).

[14] John Cotton and Monica Adya. 2018. The Impact of E-Mentoring on Information Technology Professionals. In Proceedings of the 2018 ACM SIGMIS Conference on Computers and People Research. ACM, 119-122.

[15] Kathryn H Dansky. 1996. The effect of group mentoring on career outcomes. Group \& Organization Management 21, 1 (1996), 5-21. 
[16] Munmun De Choudhury and Sushovan De. 2014. Mental health discourse on reddit: Self-disclosure, social support, and anonymity. In Eighth International AAAI Conference on Weblogs and Social Media.

[17] Bridget F Grant, Risë B Goldstein, Tulshi D Saha, S Patricia Chou, Jeesun Jung, Haitao Zhang, Roger P Pickering, W June Ruan, Sharon M Smith, Boji Huang, and others. 2015. Epidemiology of DSM-5 alcohol use disorder: results from the National Epidemiologic Survey on Alcohol and Related Conditions III. JAMA psychiatry 72, 8 (2015), 757-766.

[18] Bridget F Grant, Tulshi D Saha, W June Ruan, Risë B Goldstein, S Patricia Chou, Jeesun Jung, Haitao Zhang, Sharon M Smith, Roger P Pickering, Boji Huang, and others. 2016. Epidemiology of DSM-5 drug use disorder: results from the National Epidemiologic Survey on Alcohol and Related Conditions-III. JAMA psychiatry 73, 1 (2016), 39-47.

[19] Kathleen Margaret Griffiths, Alison L. Calear, and Michelle Banfield. 2009. Systematic review on Internet Support Groups (ISGs) and depression (1): Do ISGs reduce depressive symptoms? Journal of medical Internet research 11, 3 (2009), e40.

[20] Shefali Haldar, Sonali R Mishra, Maher Khelifi, Ari H Pollack, and Wanda Pratt. 2018. Exploring the Design of an Inpatient Peer Support Tool: Views of Adult Patients. In AMIA Annual Symposium Proceedings, Vol. 2018. American Medical Informatics Association, 1282.

[21] A Tom Horvath and Julie Yeterian. 2012. SMART Recovery: Self-empowering, science-based addiction recovery support. Journal of Groups in Addiction \& Recovery 7, 2-4 (2012), 102-117.

[22] Chunling Hu, Simon Kung, Teresa A Rummans, Matthew M Clark, and Maria I Lapid. 2014. Reducing caregiver stress with internet-based interventions: a systematic review of open-label and randomized controlled trials. Journal of the American Medical Informatics Association 22, e1 (2014), e194-e209.

[23] Keith Humphreys, Janet C Blodgett, and Todd H Wagner. 2014. Estimating the efficacy of Alcoholics Anonymous without self-selection bias: An instrumental variables re-analysis of randomized clinical trials. Alcoholism: Clinical and Experimental Research 38, 11 (2014), 2688-2694.

[24] Hsin-Liu Cindy Kao, Bo-Jhang Ho, Allen C. Lin, and Hao-Hua Chu. 2012. Phone-based gait analysis to detect alcohol usage.. In UbiComp. 661-662.

[25] Katy Kaplan, Mark S. Salzer, Phyllis Solomon, Eugene Brusilovskiy, and Pamela Cousounis. 2011. Internet peer support for individuals with psychiatric disabilities: A randomized controlled trial. Social Science \& Medicine 72, 1 (Jan. 2011), 54-62. DOI :

http://dx.doi.org/10.1016/j.socscimed.2010.09.037

[26] JB Kingree and Martie Thompson. 2011. Participation in Alcoholics Anonymous and post-treatment abstinence from alcohol and other drugs. Addictive Behaviors 36, 8 (2011), 882-885.

[27] Kathy E. Kram and Lynn A. Isabella. 1985. Mentoring alternatives: The role of peer relationships in career development. Academy of management Journal 28, 1 (1985), 110-132.

[28] Vicki L. Baker, Meghan J. Pifer, and Kimberly A. Griffin. 2014. Mentor-protégé fit: Identifying and developing effective mentorship across identities in doctoral education. International Journal for Researcher Development 5, 2 (2014), 83-98.

[29] Guo Li, Xiaomu Zhou, Tun Lu, Jiang Yang, and Ning Gu. 2016. SunForum: Understanding Depression in a Chinese Online Community. In Proceedings of the 19th ACM Conference on Computer-Supported Cooperative Work \& Social Computing. ACM, 515-526.

[30] Rachel Lipari. 2017. The CBHSQ Report. https://www. samhsa.gov/data/sites/default/files/ report_2790/ShortReport-2790.html. (june 2017).

[31] Diana MacLean, Sonal Gupta, Anna Lembke, Christopher Manning, and Jeffrey Heer. 2015. Forum77: An analysis of an online health forum dedicated to addiction recovery. In Proceedings of the 18th ACM Conference on Computer Supported Cooperative Work \& Social Computing. ACM, 1511-1526.

[32] Barbara S McCrady. 2004. To have but one true friend: implications for practice of research on alcohol use disorders and social network. Psychology of Addictive Behaviors 18, 2 (2004), 113.

[33] R Kathryn McHugh, Bridget A Hearon, and Michael W Otto. 2010. Cognitive behavioral therapy for substance use disorders. Psychiatric Clinics 33, 3 (2010), 511-525.

[34] David C. Mohr, Michelle Nicole Burns, Stephen M. Schueller, Gregory Clarke, and Michael Klinkman. 2013. Behavioral Intervention Technologies: Evidence review and recommendations for future research in mental health. General Hospital Psychiatry 35, 4 (July 2013), 332-338. DOI :

http://dx.doi .org/10.1016/j.genhosppsych.2013.03.008

[35] Robert R. Morris, Stephen M. Schueller, and Rosalind W. Picard. 2015. Efficacy of a web-based, crowdsourced peer-to-peer cognitive reappraisal platform for depression: randomized controlled trial. Journal of medical Internet research 17, 3 (2015), e72.

[36] Wendy Marcinkus Murphy. 2011. From e-mentoring to blended mentoring: increasing students' developmental initiation and mentors' satisfaction. Academy of Management Learning \& Education 10, 4 (2011), 606-622.

[37] Andrea Rae Neely, John Cotton, and Anthony David Neely. 2017. E-mentoring: A Model and Review of the Literature. AIS Transactions on Human-Computer Interaction 9, 3 (2017), 220-242. 
[38] General Service Office. 2019. Estimates of A.A. Groups and Members.

https://www.aa.org/assets/en_US/smf-53_en.pdf. (January 2019).

[39] Kathleen O'Leary, Arpita Bhattacharya, Sean A. Munson, Jacob O. Wobbrock, and Wanda Pratt. 2017. Design Opportunities for Mental Health Peer Support Technologies. In Proceedings of the 2017 ACM Conference on Computer Supported Cooperative Work and Social Computing (CSCW'17). ACM, New York, NY, USA, 1470-1484. DOI :

http://dx.doi .org/10.1145/2998181.2998349 event-place: Portland, Oregon, USA.

[40] Kathleen O'Leary, Stephen M Schueller, Jacob O Wobbrock, and Wanda Pratt. 2018a. âĂIJSuddenly, we got to become therapists for each otherâĂİ: Designing Peer Support Chats for Mental Health. In Proceedings of the 2018 CHI Conference on Human Factors in Computing Systems. ACM, 331.

[41] Kathleen O'Leary, Stephen M. Schueller, Jacob O. Wobbrock, and Wanda Pratt. 2018b. âĂIJSuddenly, We Got to Become Therapists for Each OtherâĂİ: Designing Peer Support Chats for Mental Health. In Proceedings of the 2018 CHI Conference on Human Factors in Computing Systems (CHI '18). ACM, New York, NY, USA, 331:1-331:14. DOI:

http://dx.doi.org/10.1145/3173574.3173905 event-place: Montreal QC, Canada.

[42] Judith S. Olson and Wendy A. Kellogg. 2014. Ways of Knowing in HCI. Springer Science \& Business.

[43] MARIA E. PAGANO, KAREN B. FRIEND, J. SCOTT TONIGAN, and ROBERT L. STOUT. 2004. Helping Other Alcoholics in Alcoholics Anonymous and Drinking Outcomes: Findings from Project MATCH. Journal of studies on alcohol 65, 6 (Nov. 2004), 766-773.

http://www.ncbi.nlm.nih.gov/pmc/articles/PMC3008319/

[44] Jessica A. Pater, Oliver L. Haimson, Nazanin Andalibi, and Elizabeth D. Mynatt. 2016. "Hunger Hurts but Starving Works": Characterizing the presentation of eating disorders online. In Proceedings of the 19th ACM Conference on Computer-Supported Cooperative Work \& Social Computing. ACM, 1185-1200.

[45] Ria Poole, Daniel Smith, and Sharon Simpson. 2015. How Patients Contribute to an Online Psychoeducation Forum for Bipolar Disorder: A Virtual Participant Observation Study. JMIR Mental Health 2, 3 (2015), e21. DOI : http://dx.doi.org/10.2196/mental.4123

[46] Belle Rose Ragins. 1995. Diversity, power, and mentorship in organizations: A cultural, structural, and behavioral perspective. In Diversity in organizations: New perspectives for a changing workplace. Sage Publications, Inc, Thousand Oaks, CA, US, 91-132. DOI: http://dx.doi.org/10.4135/9781452243405.n5
[47] Belle Rose Ragins and Terri A. Scandura. 1999. Burden or blessing? Expected costs and benefits of being a mentor. Journal of Organizational Behavior: The International Journal of Industrial, Occupational and Organizational Psychology and Behavior 20, 4 (1999), 493-509.

[48] Amon Rapp, Maurizio Tirassa, and Lia Tirabeni. 2019. Rethinking Technologies for Behavior Change: A View from the Inside of Human Change. ACM Transactions on Computer-Human Interaction (TOCHI) 26, 4 (2019), 22.

[49] Ralph L Rosnow and Robert Rosenthal. 1976. The volunteer subject revisited. Australian Journal of Psychology 28, 2 (1976), 97-108.

[50] Sabirat Rubya and Svetlana Yarosh. 2017a. Interpretations of online anonymity in Alcoholics Anonymous and Narcotics Anonymous. Proceedings of the ACM on Human-Computer Interaction 1, CSCW (2017), 91.

[51] Sabirat Rubya and Svetlana Yarosh. 2017b. Video-Mediated Peer Support in an Online Community for Recovery from Substance Use Disorders. In Proceedings of the 2017 ACM Conference on Computer Supported Cooperative Work and Social Computing (CSCW '17). ACM, New York, NY, USA, 1454-1469. DOI : http://dx.doi .org/10.1145/2998181.2998246 event-place: Portland, Oregon, USA.

[52] Zachary Schmitt and Svetlana Yarosh. 2018. Participatory Design of Technologies to Support Recovery from Substance Use Disorders. Proceedings of the ACM on Human-Computer Interaction 2, CSCW (2018), 156.

[53] Kate M Scott, Carmen Lim, Ali Al-Hamzawi, Jordi Alonso, Ronny Bruffaerts, José Miguel Caldas-de Almeida, Silvia Florescu, Giovanni De Girolamo, Chiyi $\mathrm{Hu}$, Peter De Jonge, and others. 2016. Association of mental disorders with subsequent chronic physical conditions: world mental health surveys from 17 countries. JAMA psychiatry 73, 2 (2016), 150-158.

[54] Narcotics Anonymous World Services. 2019. Information about NA. Brochure. (2019). "https://www.na.org/admin/include/spaw2/uploads/pdf/ PR/2302_2018.pdf"

[55] Dianna L. Stone and Kimberly M. Lukaszewski. 2009. An expanded model of the factors affecting the acceptance and effectiveness of electronic human resource management systems. Human Resource Management Review 19, 2 (2009), 134-143.

[56] Acar Tamersoy, Duen Horng Chau, and Munmun De Choudhury. 2017. Analysis of Smoking and Drinking Relapse in an Online Community. In Proceedings of the 2017 International Conference on Digital Health. ACM, 33-42. 
[57] Acar Tamersoy, Munmun De Choudhury, and Duen Horng Chau. 2015. Characterizing smoking and drinking abstinence from social media. In Proceedings of the 26th ACM Conference on Hypertext \& Social Media. ACM, 139-148.

[58] Michael Todd, Keith E. Davis, and Thomas P. Cafferty. 1984. Who Volunteers for Adult Development Research?: Research Findings and Practical Steps to Reach Low Volunteering Groups. The International Journal of Aging and Human Development 18, 3 (1984), 177-184. DOI:

http://dx. doi .org/10.2190/AYK2-QRJ7-N8PU-M9B5

[59] J. Scott Tonigan and Samara L. Rice. 2010. Is it beneficial to have an alcoholics anonymous sponsor? Psychology of Addictive Behaviors 24, 3 (2010), 397.

[60] Ellen Tuchman. 2010. Women and addiction: the importance of gender issues in substance abuse research. Journal of addictive diseases 29, 2 (2010), 127-138.

[61] Gary R VandenBos. 2007. APA dictionary of psychology. American Psychological Association.

[62] Yunlong Wang, Ahmed Fadhil, and Harald Reiterer. 2019. Health Behavior Change in HCI: Trends, Patterns, and Opportunities. arXiv preprint arXiv:1901.10449 (2019).

[63] Jane Witbrodt, Lee Kaskutas, Jason Bond, and Kevin Delucchi. 2012. Does sponsorship improve outcomes above Alcoholics Anonymous attendance? A latent class growth curve analysis. Addiction 107, 2 (2012), 301-311.

[64] Richmond Y Wong and Deirdre K Mulligan. 2019. Bringing Design to the Privacy Table: Broadening" Design" in" Privacy by Design" Through the Lens of HCI. (2019).

[65] Svetlana Yarosh. 2013. Shifting dynamics or breaking sacred traditions?: the role of technology in twelve-step fellowships. In Proceedings of the SIGCHI Conference on Human Factors in Computing Systems. ACM, 3413-3422.

[66] Chuang-Wen You, Ya-Fang Lin, Cheng-Yuan Li, Yu-Lun Tsai, Ming-Chyi Huang, Chao-Hui Lee, Hao-Chuan Wang, and Hao-Hua Chu. 2016. KeDiary:
Using Mobile Phones to Assist Patients in Recovering from Drug Addiction. In Proceedings of the $2016 \mathrm{CHI}$ Conference on Human Factors in Computing Systems. ACM, 5704-5709.

[67] Chuang-Wen You, Jui-Ting Tsai, Hung-Yeh Lin, Yu-Ting Wang, Yi-Ching Huang, Diane Lu, Yi-Ju Chung, Yaliang Chuang, Chia-Hua Kuo, Ming-Chyi Huang, Jane Yung-Jen Hsu, and Hui-Ching Wu. 2018. Using Mobile Phones to Facilitate Alcohol Dependent Patients to Improve Family Communication. In Proceedings of the 2018 ACM International Joint Conference and 2018 International Symposium on Pervasive and Ubiquitous Computing and Wearable Computers (UbiComp '18). ACM, New York, NY, USA, 311-314. DOI :

http://dx.doi .org/10.1145/3267305.3267566

[68] Chuang-wen You, Kuo-Cheng Wang, Ming-Chyi Huang, Yen-Chang Chen, Cheng-Lin Lin, Po-Shiun Ho, Hao-Chuan Wang, Polly Huang, and Hao-Hua Chu. 2015. Soberdiary: A phone-based support system for assisting recovery from alcohol dependence. In Proceedings of the 33rd Annual ACM Conference on Human Factors in Computing Systems. ACM, 3839-3848.

[69] Ye Yuan and Svetlana Yarosh. 2019. Beyond Tutoring: Opportunities for Intergenerational Mentorship at a Community Level. In Proceedings of the 2019 CHI Conference on Human Factors in Computing Systems (CHI '19). ACM, New York, NY, USA, Article 449, 14 pages. DOI : http://dx. doi .org/10.1145/3290605.3300679

[70] Shuo Zhou, Timothy Bickmore, Amy Rubin, Catherine Yeksigian, Molly Sawdy, and Steven R. Simon. 2018. User Gaze Behavior While Discussing Substance Use with a Virtual Agent. In Proceedings of the 18th International Conference on Intelligent Virtual Agents (IVA '18). ACM, New York, NY, USA, 353-354. DOI : http://dx.doi .org/10.1145/3267851.3267906

[71] William H Zywiak, Richard Longabaugh, and Philip W Wirtz. 2002. Decomposing the relationships between pretreatment social network characteristics and alcohol treatment outcome. Journal of Studies on Alcohol 63, 1 (2002), 114-121. 